Onkologie 1996;19:460-462

\title{
Contents, Vol. 19, No. 6, 1996
}

Inhalt

OMKOLOGIE

Vol. 19, Issue 6, December 1996

Vol. 19, Heft 6, Dezember 1996

Review Articles

Übersichtsarbeiten

464 The Cell Cycle - Theory and Application to Cancer

Parwaresch, R.; Rudolph, P. (Kiel)

474 Methods to Detect MDRl/P-Glycoprotein in Clinical Tumor Samples

Lehnert, M. (St. Gallen)

480 Current Insights in the Treatment of Superficial Transitional Cell Carcinoma of the Bladder Reijke de, T.M.; Kurth, K.; Boer de, E.C.; Schamhart, D.H.J. (Amsterdam)

464 Der Zellzyklus - Theorie und Anwendung in der Tumorpathologie

Parwaresch, R,; Rudolph, P. (Kiel)

474 Methoden zum Nachweis von MDRl/P-Glykoprotein in klinischen Tumorproben

Lehnert, M. (St. Gallen)

480 Neue Einblicke in die Behandlung des superfiziellen Transitionalzellkarzinoms der

Harnblase

Reijke de. T.M.; Kurth, K; Boer de, E.C.: Schamhart. D.H.J. (Amsterdam)

Original Articles

Originalarbeiten

490 Phase I Clinical and Pharmacokinetic Study of

LU103793 (Cemadotin Hydrochloride) as an Intravenous Bolus Injection in Patients with Metastatic Solid Tumors

Mross, K. (Hamburg, Freiburg); Herbst, K. (Hamburg); Berdel, W.E.; Korfel, A. (Berlin); von Broen, I.-M.; Bankmann, Y. (Ludwigshafen); Hossfeld, D.K. (Hamburg)

496 Salvage Chemotherapy with Dacarbazine or

Carboplatin/VP-16 of Advanced Soft Tissue Sarcoma Pretreated with Doxorubicin/Ifosfamide Holstein, K.; Weh, H. J,; Walter, T. A.; Hossfeld. D.K. (Hamburg)

501 Effect of Combined Natural Human Beta-Interferon and Radiation on Human Tumor Cells Busch, M. (München); Rave-Frank, M.; Franke, T. (Göttingen); Dühmke, E. (München) 490 Phase-I- und Pharmakokinetikstudie mit LU103793 (Cemadotin Hydrochlorid) als intravenöse Bolusinfektion bei Patienten mit Malignomen Mross, K. (Hamburg, Freiburg); Herbst, K. (Hamburg); Berdel, W.E.; Korfel, A. (Berlin); von Broen, I.-M.; Bankmann, Y. (Ludwigshafen); Hossfeld, D.K. (Hamburg) 
496 Einsatz von Dacarbazin oder Carboplatin/VP-16 bei fortgeschrittenen Weichteilsarkomen nach Vorbehandlung mit Doxorubicin und Ifosfamid

Holstein, K.; Weh, H.J.; Walter, T. A.; Hossfeld, D.K. (Hamburg)

501 Effekt der Kombination von natürlichem humanem Beta-Interferon und Bestrahlung auf humane Tumorzellen

Busch, M. (München); Rave-Frank, M.; Franke, T. (Göttingen); Dühmke, E. (München)

Casuistic Contributions

Kasuistiken

506 Axillary Metastatic Spread in Connection with Local Recurrence of a Renal Cell Carcinoma 22 Years after the First Diagnosis - Case Report and Survey of the Literature

Wiegel, T.; Degner, C; Comely, D.; Runkel, N. (Berlin)

508 13-Cis Retinoic Acid, Interferon-alpha and Concomittant Irradiation in a Patient with NonSmall-Cell Lung Cancer - Description of Toxicity and Response

Schiebe, M.; Hoffmann, W.; Vogel, U.; Bültmann, B.; Bamberg, M. (Tubingen)

506 Axilläre Metastasierung in Verbindung mit einem Lokalrezidiv bei einem Nierenzellkarzinom 22 Jahre nach Erstdiagnose - Kasuistik und Literaturübersicht

Wiegel, T.; Degner, C; Comely, D.; Runkel, N, (Berlin)

508 13-cis-Retinsäure und alpha-Interferon begleitend zur Strahlentherapie eines nichtkleinzelligen Bronchialkarzinoms

Schiebe, M.; Hoffmann, W,; Vogel, LI; Bültmann, B.; Bamberg, M. (Tubingen)

Clinical Information

Klinische Information

513 Guidelines for the Therapy of Esophageal Cancer

513 Leitlinien zur Therapie des Ösophaguskarzinoms

516 Guidelines for the Therapy of Exocrine Pancreatic Cancer

516 Leitlinien zur Therapie des exokrinen Pankreaskarzinoms

Continued on page 462

Fortsetzung auf Seite 462

Bibliographischer Hinweis: Inhaltsverzeichnisse dieser Zeitschrift erscheinen regelmäßig in current contents ${ }^{\circledR}$ sowie in anderen bibliographischen Diensten.

Contents a Inhalt

OHKOLOCIE

Vol. 19, Issue 6, December 1996

Vol. 19, Heft 6, Dezember 1996

520 Book Reviews

520 Buchbesprechungen

524 Obituary 
524 Nachruf

525 Industrial Forum

525 Industrieforum

526 Reports of Oncological Societies

526 Mitteilungen onkologischer Gesellschaften

459 Imprint

527 Author Index 1996

531 Subject Index 1996

VII Complete Contents 1996 (following page 532)

U2 Instructions to Authors (inside front cover)

459 Impressum

527 Autorenverzeichnis 1996

529 Sachwortverzeichnis 1996

III Jahres-Inhalt 1996 (nach Seite 532)

U3 Hinweise für Autoren (3. Umschiagseite)

Contents of Next Issues

Original Article

Locoregional Chemotherapy of Gynecological Cancer

Baltzer, J.; Poleska, W.; Fiedler, V. (Krefeld)

Review Article

Ibandronate in Malignant Bone Disease

Scigalla, P. (Mannheim)

Short Review

Does Reconstructive Breast Surgery and Breast Conserving Therapy Improve the Quality of

Life?

Neises, M. (Mannheim)

Oncological Education Diffuse Malignant Pleural Mesothelioma in Diagnosis, Therapy, and Prognosis: A Review

Manegold, C; Lutz, D.; Schirren, I; Schneider, P.; Schraube, P.; Drings, P.; Vogt-Moykopf, I.;

Wannenmacher, M. (Heidelberg)

Clinical Information

Hygienic Problems with Mineralwater

Daschner. F. (Freiburg) 